\title{
Dynamics of Nonautonomous Stochastic Gilpin-Ayala Competition Model with Jumps
}

\author{
Yanchao Zang, ${ }^{1}$ Junping $L i,{ }^{1}$ and Jiangang $\operatorname{Liu}^{2}$ \\ ${ }^{1}$ School of Mathematics and Statistics, Central South University, Changsha 410075, China \\ ${ }^{2}$ School of Information Science and Engineering, Central South University, Changsha 410075, China \\ Correspondence should be addressed to Yanchao Zang; zycmail@126.com
}

Received 20 May 2013; Revised 14 September 2013; Accepted 19 September 2013

Academic Editor: Chun-Lei Tang

Copyright (C) 2013 Yanchao Zang et al. This is an open access article distributed under the Creative Commons Attribution License, which permits unrestricted use, distribution, and reproduction in any medium, provided the original work is properly cited.

\begin{abstract}
The nonautonomous stochastic Gilpin-Ayala competition model driven by Lévy noise is considered. First, it is shown that this model has a global positive solution. Then, we discuss the asymptotic behavior of the solution including moment and pathwise estimation. Finally, sufficient conditions for extinction, nonpersistence in the mean, and weak persistence of the solution are established.
\end{abstract}

\section{Introduction}

Population dynamics that describes and understands changes in populations over time is an important subfield of ecology. One of the famous models for population dynamics is the traditional Lotka-Volterra competition system which has received a lot of attention and has been studied extensively because of its theoretical and practical significance. It was suggested by Lotka [1] and Volterra [2] in the 1920s and was described by

$$
\dot{x}_{i}(t)=x_{i}(t)\left[r_{i}(t)-\sum_{j=1}^{n} a_{i j}(t) x_{j}(t)\right], \quad i=1,2, \ldots, n,
$$

where $x_{i}(t)$ represents the population size of species $i$ at time $t, r_{i}(t)$ is the rate of growth at time $t, a_{i j}(t)$ represents the effect of interspecies or intraspecies at time $t$, and $r_{i}(t) / a_{i j}(t)$ is the carrying capacity of the $i$ th species in the absence of other species at time $t$. There is an extensive literature concerned with the dynamics of (1); see, for example, Gopalsamy [3], Kuang [4], Liao and Tang [5], and Xiao and Li [6]. In particular, the books by Gopalsamy [3] and Kuang [4] are good references in this area. However, the Lotka-Volterra model has often been severely criticized. One of the criticisms is that in such a model the rate of change in the density of species is a linear function of densities of the interacting species. Hence, in order to yield significantly more accurate and realistic results, in 1973, Gilpin and Ayala [7] proposed a more complicated model as follows:

$$
\begin{array}{r}
\dot{x}_{i}(t)=r_{i} x_{i}(t) \\
\times\left[1-\left(\frac{x_{i}(t)}{K_{i}}\right)^{\theta_{i}}-\sum_{j=1, j \neq i}^{n} b_{i j}(t) \frac{x_{j}(t)}{K_{j}}\right], \\
i=1,2, \ldots, n,
\end{array}
$$

where $\theta_{i}$ are the parameters to modify the classical LotkaVolterra model. Since then, some related research has appeared in the literature [8]. And $\mathrm{He}$ and his coauthors in [9] considered Gilpin-Ayala competition system with impulses; they investigated some properties, such as permanence, extinction, and global attraction, of the determined Gilpin-Ayala competition system. They mainly considered the impulses effects on the long behavior of the system.

On the other hand, population dynamics in the real world is inevitably affected by environment noise which is an important component in an ecosystem. So it is necessary and important to consider the corresponding stochastic population model. For the stochastic population models, we refer the reader to [10-13] mainly dealing with Lotka-Volterra model and [14-16] mainly dealing with stochastic GilpinAyala models. Furthermore, the population may suffer from 
sudden environmental shocks, for example, earthquakes, hurricanes, epidemics, and so forth. However, the above mentioned stochastic models cannot explain such phenomena. To explain these phenomena, we introduce a jump process into the stochastic population dynamics. As far as we know, there are few papers considering the stochastic population models with jumps $[10,17,18]$. However, still no scholars investigated the nonautonomous stochastic GilpinAyala competitive model with Poisson jumps or Lévy jumps. Zhang and Wang [18] discussed the asymptotic behavior of stochastic Gilpin-Ayala mutualism model with jumps:

$$
\begin{array}{r}
d x_{i}(t)=x_{i}(t)\left\{\left(r_{i}-a_{i i} x_{i}^{\theta_{i}}(t)-\sum_{j \neq i}^{d} a_{i j} x_{j}(t)\right) d t\right. \\
\left.+\sum_{j=1}^{m} \sigma_{i j} d B_{j}(t)+\int_{\mathbb{V}} \gamma_{i}(u) \widetilde{N}(d t, d u)\right\}, \\
i=1, \ldots, n,
\end{array}
$$

where the intrinsic exponential growth rate $r_{i}$ and the effects of interspecific (for $i \neq j$ ) and intraspecific (for $i=j$ ) interaction $a_{i j}$ are constants. In this model, some asymptotic property was obtained, especially, and the explicit solution was obtained in one-dimension case. However, in [18] it assumed that all parameters are constants in time; they are naturally subject to fluctuation in time; if a model which takes into account such fluctuations is desired, it must be nonautonomous.

Hence, motivated by the above discussion, in this paper, we consider the nonautonomous stochastic Gilpin-Ayala competition models perturbed by Lévy noise; that is,

$$
\begin{array}{r}
d x_{i}(t)=x_{i}(t)\left\{\left(r_{i}(t)-\sum_{j=1}^{n} a_{i j}(t) x_{j}^{\alpha_{i j}}(t)\right) d t\right. \\
+\sum_{j=1}^{n} \sigma_{i j}(t) x_{j}^{\theta_{i j}}(t) d w_{j}(t) \\
\left.+\int_{\mathbb{Y}} H_{i}(x(t-), u) \widetilde{N}(d t, d u)\right\}, \\
i=1, \ldots, n,
\end{array}
$$

where $w(t)=\left(w_{1}(t), \ldots, w_{n}(t)\right)^{\top}$ is a $n$-dimensional Brownian motion defined on the filtered probability space. $x_{i}(t-):=$ $\lim _{s \uparrow t} x_{i}(s), N(d t, d u)$ is a real-valued Poisson counting measure with characteristic measure $\lambda$ on a measurable subset $\mathbb{Y}$ of $[0,+\infty)$ with $\lambda(\mathbb{Y})<\infty, \widetilde{N}(d t, d u):=N(d t, d u)-\lambda(d u) d t$. $r_{i}(t)$ is the intrinsic exponential growth rate for the $i$ th species at time $t . a_{i j}(t)$ represent the effects of interspecific and intraspecific interaction at time $t ; \alpha_{i j}$ and $\theta_{i j}$ are nonnegative constants. Functions $r_{i}(t), a_{i j}(t)$, and $\sigma_{i j}(t)$ are continuous, bounded, and nonnegative on $[0,+\infty) ; H_{i}: \mathbb{R}^{n} \times \mathbb{Y} \rightarrow \mathbb{R}^{n}$ are measurable functions.
It is worth observing that the model (4) can be seen as the generalization of model (3), because it can be reduced to model (3) when $r_{i}(t) \equiv r_{i}, a_{i j}(t) \equiv a_{i j}, \sigma_{i j}(t) \equiv \sigma_{i j}, \alpha_{i j} \equiv$ $1(i \neq j), \theta_{i j} \equiv 0$, and $H_{i}(x, u)=\gamma_{i}(u), x \in \mathbb{R}_{+}^{n}$, respectively.

Throughout this paper, let $(\Omega, \mathscr{F}, P)$ be a complete probability space with a filtration $\left\{\mathscr{F}_{t}\right\}_{t \geq 0}$ satisfying the usual conditions; that is, it is increasing and right continuous while $\mathscr{F}_{0}$ contains all $P$-null sets, and let $\mathbb{R}_{+}^{n}=\left\{x \in \mathbb{R}^{n}: x_{i}>\right.$ $0, \forall i=1, \ldots, n\}$, and $|\cdot|$ denotes the Euclidian norm in $\mathbb{R}^{n}$.

If $f(t)$ is a continuous and bounded function on $[0,+\infty)$, then define

$$
f^{u}=\sup _{t \in[0,+\infty)} f(t), \quad f^{l}=\inf _{t \in[0,+\infty)} f(t) .
$$

For any constant sequence $\left\{c_{i j}\right\}(i, j=1, \ldots, n)$, define

$$
\begin{array}{ll}
\left(\bar{c}_{i j}\right)=\max _{1 \leq i, j \leq n} c_{i j}, & \left(\bar{c}_{i i}\right)=\max _{1 \leq i \leq n} c_{i i}, \\
\left(\widetilde{c}_{i j}\right)=\min _{1 \leq i, j \leq n} c_{i j}, & \left(\widetilde{c}_{i i}\right)=\min _{1 \leq i \leq n} c_{i i} .
\end{array}
$$

The rest of the paper is arranged as follows. In Section 2, we prove that the solution of system (4) is global and positive and it does not explode at a finite time. In Section 3, we analyze the asymptotic moment behavior of the solution. The other different properties of the solution, asymptotic pathwise estimation, extinction, nonpersistence, and weak persistence, are obtained in Sections 4 and 5, respectively. In Section 6, we construct a numerical example with some figures to illustrate our main results. At last, we close the paper with conclusions in Section 7.

\section{Global Positive Solutions}

The solution of (4) $x_{i}(t)$ denotes the population sizes of $i$ th species at time $t$; it is natural to require it to be positive. Moreover, in order for a stochastic differential equation to have a unique global (i.e., no explosion in a finite time) solution for any given initial value, the coefficients of the equation are generally required to satisfy the linear growth condition and local Lipschitz condition (see, e.g., [19]). However, the coefficients of system (4) are locally Lipschitz continuous but do not satisfy the linear growth condition, so the solution of system (4) may explode at a finite time. Under some simple hypothesis, the following theorem shows that this solution is positive and global.

In what follows we always denote by $K>0$ a generic constant whose values may vary for its different appearances. To show the main result let us recall the following facts.

Consider a $n$-dimensional jump-diffusion process $x(t)$ satisfying

$$
\begin{aligned}
d x(t)= & F(x(t)) d t+G(x(t)) d w(t) \\
& +\int_{\mathbb{Y}} \Phi(x(t-), u) \widetilde{N}(d t, d u), \quad t \geq 0,
\end{aligned}
$$

with initial condition $x(0)=x_{0} \in \mathbb{R}^{n}$, where $F: \mathbb{R}^{n} \rightarrow \mathbb{R}^{n}$ and $\Phi: \mathbb{R}^{n} \times \mathbb{Y} \rightarrow \mathbb{R}^{n}$ are measurable functions and $G(x)$ 
is a $n \times m$-matrix-valued function. Here $w(t)$ is $\mathbb{R}^{m}$-valued Brownian motion, and

$$
\widetilde{N}(d t, d u)=N(d t, d u)-\lambda(d u) d t
$$

is a compensated Poisson random measure on $[0,+\infty) \times \mathbb{R}^{n}$ which is independent of $w(t)$.

The following conclusion is given by [20, Lemma 2].

Lemma 1. Let $F(\mathbf{0})=\Phi(\mathbf{0}, u)=\mathbf{0} \in \mathbb{R}^{n}, G(\mathbf{0})=\mathbf{0}$ which is a zero matrix, and $F, G$, and $\Phi$ satisfy locally Lipschitz condition. Set

$$
J(x):=\int_{\mathbb{V}}\left(\ln \frac{|x+\Phi(x, u)|}{|x|}\right)^{2} \lambda(d u), \quad x \neq 0 .
$$

If

$$
\sup _{0<|x| \leq m} J(x)<\infty, \quad \text { for each } m \geq 1 \text {, }
$$

then, for $x_{0} \neq 0$,

$$
P\left\{x\left(t, x_{0}\right) \neq 0, x\left(t-, x_{0}\right) \neq 0 \text { for any } t \geq 0\right\}=1 \text {, }
$$

where $x\left(t, x_{0}\right)$ denotes the solution of (7) starting from $x_{0}$ at time $t=0$.

Before stating the main results in this paper, we give the following assumptions for the jump-diffusion coefficient.

(H1) Assume that, for any $m \geq 1, x \in \mathbb{R}^{n}, u \in \mathbb{Y}$, and $i=1, \ldots, n$,

$$
\begin{gathered}
H_{i}(x, u)>-1, \quad H_{i}(0, u)=0, \\
\sup _{0<|x| \leq m} \int_{\mathbb{V}}\left(\ln \left|1+H_{i}(x, u)\right|\right)^{2} \lambda(d u)<\infty,
\end{gathered}
$$

and for each $k>0$ there exists $L_{k}>0$ such that

$$
\int_{\mathbb{V}}|H(x, u)-H(y, u)|^{2} \lambda(d u) \leq L_{k}|x-y|^{2}
$$

whenever $x, y \in \mathbb{R}^{n}$ with $|x| \vee|y| \leq k$.

(H2) Assume further that for $\gamma \in(0,1)$ there exist constants $\eta>0$ and $\theta>0$ such that, for $x \in \mathbb{R}^{n}, i=1, \ldots, n$,

$$
\begin{aligned}
& \int_{\mathbb{Y}}\left[\left(1+H_{i}(x, u)\right)^{\gamma}-1-\gamma H_{i}(x, u)\right] \lambda(d u) \\
& \leq-\eta|x|^{\theta}+o\left(|x|^{\theta}\right),
\end{aligned}
$$

where $o\left(|x|^{\theta}\right) /|x|^{\theta} \rightarrow 0$ as $|x| \rightarrow \infty$.

Theorem 2. Let the assumptions (H1) and (H2) hold. Then, for any given initial value $x_{0} \in \mathbb{R}_{+}^{n}$, (4) has a unique global solution $x(t) \in \mathbb{R}_{+}^{n}$ for any $t \geq 0$ a.s.

Proof. Since, by (14), the coefficients of drift and diffusion are locally Lipschitz continuous, hence, for any given initial value $x_{0} \in \mathbb{R}_{+}^{n}$, there is a unique local solution $x(t)$ on $t \in\left[0, \tau_{e}\right)$, where $\tau_{e}$ is the explosion time. By (4) we can get that

$$
\begin{gathered}
x_{i}(t) \\
=x_{i}(0) \exp \left\{\int _ { 0 } ^ { t } \left(r_{i}(s)-\sum_{j=1}^{n} a_{i j}(s) x_{j}^{\alpha_{i j}}(s)\right.\right. \\
-\frac{1}{2} \sum_{j}^{n} \sigma_{i j}^{2}(s) x_{j}^{2 \theta_{i j}}(s) \\
+\int_{\mathbb{Y}}\left[\ln \left(1+H_{i}(x(s), u)\right)\right. \\
+\int_{0}^{t} \sum_{j=1}^{n} \sigma_{i j}(s) x_{j}^{\theta_{i j}}(s) d w_{j}(s) \\
\left.+\int_{0}^{t} \int_{\mathbb{Y}} \ln \left(1+H_{i}(x(s), u)\right] \lambda(d u)\right) d s
\end{gathered}
$$

This, together with $x_{0} \in \mathbb{R}_{+}^{n}$, yields that $x_{i}(t) \geq 0$ for any $t \in\left[0, \tau_{e}\right)$. On the other hand, due to (13), for (4) the condition (10) holds. Thus according to Lemma 1, we immediately get that, for any $t \in\left[0, \tau_{e}\right), x_{i}(t)>0$ a.s. Next, to show that this solution is global, we need to show that $\tau_{e}=\infty$ a.s. Let $k_{0}>$ 0 be sufficiently large for every component of $x_{0}$ such that $\left|x_{0}\right| \leq k_{0}$. For each integer $k>k_{0}$, define the stopping time

$$
\tau_{k}=\inf \left\{t \in\left[0, \tau_{e}\right):|x(t)|>k\right\},
$$

where throughout this paper we set $\inf \emptyset=\infty$ (as usual $\emptyset$ denotes the empty set). Clearly, $\tau_{k}$ is increasing as $k \rightarrow \infty$. Set $\tau_{\infty}=\lim _{k \rightarrow \infty} \tau_{k}$, whence $\tau_{\infty} \leq \tau_{e}$ a.s. If we can show that $\tau_{\infty}=\infty$ a.s., then $\tau_{e}=\infty$ a.s., and $x(t) \in \mathbb{R}_{+}^{n}$ a.s., for all $t \geq 0$. In other words, to complete the proof all we need to show is that $\tau_{\infty}=\infty$ a.s. To show this statement, we define nonnegative $C^{2}$-function $V: \mathbb{R}_{+}^{n} \rightarrow \mathbb{R}_{+}$

$$
V(x)=\sum_{i=1}^{n} x_{i}^{\gamma}, \quad x \in \mathbb{R}_{+}^{n}
$$

where $0<\gamma<1$. Let $k>k_{0}$ and $T>0$ be arbitrary. For any $0 \leq t \leq \tau_{k} \wedge T$, we apply Itô's formula to $V(x(t))$ and obtain

$$
\begin{aligned}
d V(x(t))= & \mathscr{L} V(x(t)) d t+\gamma \sum_{i=1}^{n} x_{i}^{\gamma}(t) \\
& \times \sum_{j=1}^{n} \sigma_{i j}(t) x_{j}^{\theta_{i j}}(t) d w_{j}(t) \\
+ & \sum_{i=1}^{n} \int_{\mathbb{V}}\left[\left(1+H_{i}(x(t-), u)\right)^{\gamma}-1\right] \\
& \times x_{i}^{\gamma}(t) \widetilde{N}(d t, d u),
\end{aligned}
$$


where, for $x \in \mathbb{R}_{+}^{n}$,

$$
\begin{aligned}
& \mathscr{L} V(x(t)):= \gamma \sum_{i=1}^{n}\left[x_{i}^{\gamma}(t)\left(r_{i}(t)-\sum_{j=1}^{n} a_{i j}(t) x_{j}^{\alpha_{i j}}(t)\right)\right. \\
&\left.-\frac{(1-\gamma)}{2} x_{i}^{\gamma}(t) \sum_{j=1}^{n} \sigma_{i j}^{2}(t) x_{j}^{2 \theta_{i j}}(t)\right] \\
&+ \sum_{i=1}^{n} x_{i}^{\gamma}(t) \int_{\mathbb{V}}\left[\left(1+H_{i}(x(t), u)\right)^{\gamma}\right. \\
&\left.\quad-1-\gamma H_{i}(x(t), u)\right] \lambda(d u) \\
&:=
\end{aligned}
$$

It is not difficult to estimate

$$
\begin{aligned}
& I_{1}(x, \gamma) \\
& \leq \gamma\left[\left(\bar{r}_{i}^{u}\right) \cdot \sum_{i=1}^{n} x_{i}^{\gamma}(t)-\left(\widetilde{a}_{i i}^{l}\right)\right. \\
& \left.\quad \cdot \sum_{i=1}^{n} x_{i}^{\gamma+\alpha_{i i}}(t)-\frac{1-\gamma}{2}\left(\widetilde{\sigma}_{i i}^{l}\right)^{2} \sum_{j=1}^{n} x_{i}^{\gamma+2 \theta_{i i}}(t)\right] .
\end{aligned}
$$

On the other hand, using (15) and knowing that

$$
n^{(1-p / 2) \wedge 0}|x|^{p} \leq \sum_{i=1}^{n} x_{i}^{p} \leq n^{(1-p / 2) \vee 0}|x|^{p},
$$

for any $p>0, x \in \mathbb{R}_{+}^{n}$, we deduce

$$
I_{2}(x, \gamma) \leq-\eta|x|^{\theta+\gamma}+o\left(|x|^{\theta+\gamma}\right) .
$$

Thus, for $\theta>0$,

$$
\mathscr{L} V(x(t)) \leq K .
$$

Therefore

$$
\begin{aligned}
& d V(x(t)) \\
& \leq \quad K d t+\gamma \sum_{i=1}^{n} x_{i}^{\gamma}(t) \sum_{j=1}^{n} \sigma_{i j}(t) x_{j}^{\theta_{i j}}(t) d w_{j}(t) \\
& \quad+\sum_{i=1}^{n} \int_{\mathbb{V}}\left[\left(1+H_{i}(x(t-), u)\right)^{\gamma}-1\right] x_{i}^{\gamma}(t) \widetilde{N}(d t, d u) .
\end{aligned}
$$

Integrating the last inequality from 0 to $\tau_{k} \wedge T$ and then taking expectations yield

$$
\begin{aligned}
E V\left(x\left(\tau_{k} \wedge T\right)\right) & \leq V(x(0))+K E\left(\tau_{k} \wedge T\right) \\
& \leq V(x(0))+K T .
\end{aligned}
$$

For each $r>0$, define

$$
\mu(r):=\inf \{V(x),|x| \geq r\} .
$$

Recalling the inequality (22), it is easy to see that

$$
\lim _{r \rightarrow \infty} \mu(r)=\infty .
$$

Then we obtain that, for some constant $K>0$,

$$
\begin{aligned}
\mu(k) P\left(\tau_{k} \leq T\right) & \leq E\left(V\left(x\left(\tau_{k}\right)\right) I_{\left\{\tau_{k} \leq T\right\}}\right) \\
& \leq E V\left(x\left(\tau_{k} \wedge T\right)\right) \leq K .
\end{aligned}
$$

From this inequality, we immediately get $P\left(\tau_{\infty} \leq T\right)=0$. Since $T>0$ is arbitrary, we must have

$$
P\left(\tau_{\infty}<\infty\right)=0, \quad P\left(\tau_{\infty}=\infty\right)=1,
$$

which completes the proof.

\section{Asymptotic Moment Estimation}

In the previous section, we show that the solution of (4) is positive and will not explode in any finite time. This nice positive property allows us to further discuss asymptotic moment estimation for the solution of (4).

Theorem 3. Let conditions of Theorem 2 hold. Then, for any initial data $x_{0} \in \mathbb{R}_{+}^{n}$ and $p \in(0,1)$, there exists a constant $K$, such that

$$
\limsup _{t \rightarrow \infty} E \sum_{i=1}^{n} x_{i}^{p}(t) \leq K .
$$

Proof. For any $p \in(0,1)$, let $V(x)=\sum_{i=1}^{n} x_{i}^{p}$ as in Theorem 2 . For any $\left|x_{0}\right|<k$, define a stopping time

$$
\sigma_{k}:=\inf \{t \geq 0:|x(t)|>k\} .
$$

From the argument of Theorem 2, we have $\sigma_{k}<\infty$ and $\sigma_{k} \uparrow$ $\infty$ a.s. as $k \rightarrow \infty$. Using Itô's formula to $e^{t} V(x)$ implies

$$
d\left(e^{t} V(x(t))\right) \leq e^{t} F(x(t)) d t
$$

$$
\begin{gathered}
+p e^{t} \sum_{i, j=1}^{n} \sigma_{i j}(t) x_{i}^{p}(t) x_{j}^{\theta_{i j}}(t) d w_{j}(t) \\
+e^{t} \sum_{i=1}^{n} \int_{\mathbb{V}}\left[\left(1+H_{i}(x(t-), u)\right)^{p}-1\right] \\
\times x_{i}^{p}(t) \widetilde{N}(d t, d u),
\end{gathered}
$$

where

$$
\begin{aligned}
& F(x(t)) \\
& \begin{aligned}
\leq p \sum_{i=1}^{n} x_{i}^{p}(t)\left[\frac{1}{p}\right. & +\left(\bar{r}_{i}^{u}\right)-\left(\widetilde{a}_{i j}^{l}\right) \sum_{j=1}^{n} x_{j}^{\alpha_{i j}}(t) \\
& \left.\quad-\frac{1-p}{2}\left(\widetilde{\sigma}_{i j}^{u}\right)^{2} \sum_{j=1}^{n} x_{j}^{2 \theta_{i j}}(t)-\eta|x|^{\theta}\right] .
\end{aligned}
\end{aligned}
$$


Obviously, for $\eta>0$, there exists a positive constant $K$ such that $F(x(t)) \leq K$, and we have

$$
\begin{gathered}
d\left(e^{t} V(x(t))\right) \\
\leq e^{t} K d t+p e^{t} \sum_{i, j=1}^{n} \sigma_{i j}(t) x_{i}^{p}(t) x_{j}^{\theta_{i j}}(t) d w_{j}(t) \\
+e^{t} \sum_{i=1}^{n} \int_{\mathbb{Y}}\left[\left(1+H_{i}(x(t-), u)\right)^{p}-1\right] \\
\times x_{i}^{p}(t) \widetilde{N}(d t, d u) .
\end{gathered}
$$

Integrating 0 into $t \wedge \sigma_{k}$ and taking expectation give

$$
E\left(e^{t \wedge \sigma_{k}} V\left(x\left(t \wedge \sigma_{k}\right)\right)\right) \leq V(x(0))+K\left(E e^{t \wedge \sigma_{k}}-1\right) .
$$

Hence, letting $k \rightarrow \infty$, we get

$$
E\left(e^{t} V(x(t))\right) \leq K\left(1+e^{t}\right)
$$

and the desired conclusion follows.

If we note the inequality

$$
\begin{aligned}
E|x(t)|^{p} & =E\left(\sum_{i=1}^{n} x_{i}^{2}(t)\right)^{p / 2} \\
& \leq E\left(d \max _{1 \leq i \leq n} x_{i}^{2}(t)\right)^{p / 2} \leq d^{p / 2} E \sum_{i=1}^{n} x_{i}^{p}(t),
\end{aligned}
$$

we claim that, for any $p \in(0,1)$, $p$ th moment of the solution of system (4) is also asymptotically bounded, which is stated in the following corollary.

Corollary 4. Under the conditions of Theorem 2, for any initial $x_{0} \in \mathbb{R}_{+}^{n}$ and $p \in(0,1)$, the solution to (4) obeys

$$
\limsup _{t \rightarrow \infty} E|x(t)|^{p} \leq K
$$

In the following theorem, we prove that the average in time of the moment of the solution to (4) is bounded.

Theorem 5. Under the conditions of Theorem 2, for any $p \in$ $(0,1)$ and any initial value $x_{0} \in \mathbb{R}_{+}^{n}$, consider the following.

(1) If $\left(\vec{a}_{i i}^{l}\right)>0$, then

$$
\limsup _{t \rightarrow \infty} \frac{1}{t} \int_{0}^{t} E|x(s)|^{p+\alpha_{i j}} d s \leq K
$$

(2) If $\left(\widetilde{\sigma}_{i i}^{l}\right)>0$, then

$$
\limsup _{t \rightarrow \infty} \frac{1}{t} \int_{0}^{t} E|x(s)|^{p+2 \theta_{i j}} d s \leq K
$$

Proof. For $0<p<1$, using the same Lyapunov function $V(x)=\sum_{i=1}^{n} x_{i}^{p}$ and by (19) and (20), we get

$$
\begin{aligned}
& d V(x(t)) \leq p\left[\left(\bar{r}_{i}^{u}\right) \cdot \sum_{i=1}^{n} x_{i}^{p}(t)-\left(\vec{a}_{i i}^{l}\right)\right. \\
& \cdot \sum_{i=1}^{n} x_{i}^{p+\alpha_{i i}}(t)-\frac{1-p}{2}\left(\widetilde{\sigma}_{i i}^{l}\right)^{2} \sum_{i=1}^{n} x^{p+1} \\
&+ \sum_{i=1}^{n} x_{i}^{p}(t) \int_{\mathbb{V}}\left[\left(1+H_{i}(x(t), u)\right)^{p}\right. \\
&+ p \sum_{i=1}^{n} x_{i}^{p}(t) \sum_{j=1}^{n} \sigma_{i j}(t) x_{j}^{\theta_{i j}}(t) d w_{j}(t) \\
&+ \sum_{i=1}^{n} \int_{\mathbb{Y}}\left[\left(1+H_{i}(x(t-), u)\right)^{p}-1\right] \\
& \times x_{i}^{p}(t) \widetilde{N}(d t, d u) .
\end{aligned}
$$$$
\left.\cdot \sum_{i=1}^{n} x_{i}^{p+\alpha_{i i}}(t)-\frac{1-p}{2}\left(\widetilde{\sigma}_{i i}^{l}\right)^{2} \sum_{i=1}^{n} x_{i}^{p+2 \theta_{i i}}(t)\right] d t
$$$$
\left.-1-p H_{i}(x(t), u)\right] \lambda(d u) d t
$$

According to (15) of the assumption (H2), we conclude that the term

$$
\begin{aligned}
& p\left(\bar{r}_{i}^{u}\right) \cdot \sum_{i=1}^{n} x_{i}^{p}(t) \\
& +\sum_{i=1}^{n} x_{i}^{p}(t) \int_{\mathbb{Y}}\left[\left(1+H_{i}(x(t), u)\right)^{p}-1-p H_{i}(x(t), u)\right] \lambda(d u)
\end{aligned}
$$

has an upper positive bound $K$ (dependent on $p$ ). Therefore for Case (1),

$$
\begin{aligned}
& V(x(t))+p\left(\tilde{a}_{i i}^{l}\right) \int_{0}^{t} \sum_{i=1}^{n} x_{i}^{p+\alpha_{i i}}(s) d s \\
& \quad \leq V(x(0))+K t+M_{1}(t)+M_{2}(t),
\end{aligned}
$$

where

$$
\begin{gathered}
M_{1}(t)=\int_{0}^{t} p \sum_{i=1}^{n} x_{i}^{p}(s) \sum_{j=1}^{n} \sigma_{i j}(s) x_{j}^{\theta_{i j}}(s) d w_{j}(s), \\
M_{2}(t)=\sum_{i=1}^{n} \int_{0}^{t} \int_{\mathbb{Y}}\left[\left(1+H_{i}(x(s-), u)\right)^{p}-1\right] \\
\times x_{i}^{p}(s) \widetilde{N}(d s, d u)
\end{gathered}
$$

are two local martingales vanishing at $t=0$. Taking expectations on both sides of (44) gives

$$
E\left[\int_{0}^{t} \sum_{i=1}^{n} x_{i}^{p+\alpha_{i i}}(s) d s\right] \leq \frac{V(x(0))+K t}{p\left(\tilde{a}_{i i}^{l}\right)} .
$$

Hence, dividing by $t$ on both sides and from inequality (38), Case (1) follows immediately. 
Case (2): if $\left(\widetilde{\sigma}_{i i}^{l}\right)>0$, we can also get that

$$
\begin{gathered}
V(x(t))+\frac{p(1-p)}{2}\left(\widetilde{\sigma}_{i i}^{l}\right)^{2} \int_{0}^{t} \sum_{i=1}^{n} x_{i}^{p+2 \theta_{i i}}(s) d s \\
\leq V(x(0))+K t+M_{1}(t)+M_{2}(t),
\end{gathered}
$$

and taking expectations on both sides yields

$$
E\left[\int_{0}^{t} \sum_{i=1}^{n} x_{i}^{p+2 \theta_{i i}}(s) d s\right] \leq \frac{2(V(x(0))+K t)}{p(1-p)\left(\widetilde{\sigma}_{i i}^{l}\right)^{2}} .
$$

Hence, the assertion of Case (2) follows as before.

\section{Pathwise Estimation}

In the previous section we have discussed how the solution of system (4) varies in $\mathbb{R}_{+}^{n}$ in moment. In this section we examine pathwise properties of the solution of system (4); that is, we consider some limit inequalities for growth rates of the population size of species, which shows how the solution of system (4) varies in $\mathbb{R}_{+}^{n}$. First, we need the following exponential martingale inequality with jumps [21, Theorem 5.2.9] to prove the main result in this part.

Lemma 6. Assume that $g:[0, \infty) \rightarrow \mathbb{R}$ and $h:[0, \infty) \times$

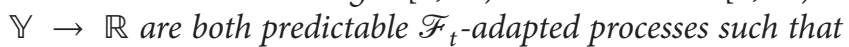
for any $T>0$

$$
\begin{gathered}
\int_{0}^{T}|g(t)|^{2} d t<\infty \quad \text { a.s., } \\
\int_{0}^{T} \int_{\mathbb{Y}}|h(t, u)|^{2} \lambda(d u) d t<\infty \quad \text { a.s. }
\end{gathered}
$$

Then for any constants $\alpha, \beta>0$

$$
P\left(\sup _{0 \leq t \leq T} Y_{\alpha}(t)>\beta\right) \leq e^{-\alpha \beta}
$$

where

$$
\begin{aligned}
Y_{\alpha}(t)= & Y(t)-\frac{\alpha}{2} \int_{0}^{t}|g(s)|^{2} d s \\
& -\frac{1}{\alpha} \int_{0}^{t} \int_{\mathbb{Y}}\left[e^{\alpha h(s, u)}-1-\alpha h(s, u)\right] \lambda(d u) d s, \\
Y(t)= & \int_{0}^{t} g(s) d W(s)+\int_{0}^{t} \int_{\mathbb{Y}} h(s, u) \widetilde{N}(d s, d u) .
\end{aligned}
$$

Theorem 7. Let the conditions of Theorem 2 hold. Assume further that there exists a constant $\delta \in\left(0, \alpha_{i i} \wedge 2 \theta_{i i}\right]$ such that

$$
|H(x, u)| \leq K|x|^{\delta}+o\left(|x|^{\delta}\right) .
$$

If $\left(\widetilde{a}_{i i}^{l}\right)>0$ or $\left(\widetilde{\sigma}_{i i}^{l}\right)>0$, then there exists $K>0$, independent of initial value $x_{0} \in \mathbb{R}_{+}^{n}$, such that the solution $x(t), t \geq 0$, of (4) has the property

$$
\limsup _{t \rightarrow \infty} \frac{\ln (|x(t)|)}{\ln t} \leq K \quad \text { a.s. }
$$

Proof. Let $U(x)=\sum_{i=1}^{n} x_{i}$ be the Lyapunov function; for convenience, we denote $Q(x, u):=\sum_{i=1}^{n} x_{i}\left(1+H_{i}(x, u)\right) / \sum_{i=1}^{n} x_{i}$ and $Z(t):=\sum_{j=1}^{n}\left(\sum_{i=1}^{n} \sigma_{i j}(s) x_{i}(t) x_{j}^{\theta_{i j}}(t)\right)^{2}$. Applying Itô's formula to $e^{t} \ln U(x)$ yields

$$
\begin{aligned}
& d\left[e^{t} \ln U(x(t))\right] \\
& \leq e^{t}[\ln U(x(t)) \\
& +\frac{\sum_{i=1}^{n} x_{i}(t)\left(r_{i}(t)-\sum_{j=1}^{n} a_{i j}(t) x_{j}^{\alpha_{i j}}(t)\right)}{U(x(t))} \\
& +e^{t} \int_{\mathbb{Y}}[\ln Q(x(t), u)-Q(x(t), u)+1] \lambda(d u) d t \\
& +\frac{e^{t}}{U(x(t))} \sum_{i, j=1}^{n} \sigma_{i j}(t) x_{i}(t) x_{j}^{\theta_{i j}}(t) d w_{j}(t) \\
& +e^{t} \int_{\mathbb{Y}} \ln Q(x(t-), u) \widetilde{N}(d t, d u) .
\end{aligned}
$$

Integrating 0 into $t$ yields

$$
\begin{gathered}
e^{t} \ln U(x(t)) \leq \ln U(x(0))+M_{1}(t)+M_{2}(t) \\
+\int_{0}^{t} e^{s}\left[\ln U(x(s))+\frac{1}{U(x(s))} \sum_{i=1}^{n} x_{i}(s) r_{i}(s)\right. \\
\quad-\frac{1}{U(x(s))} \sum_{i, j=1}^{n} x_{i}(s) a_{i j}(s) x_{j}^{\alpha_{i j}}(s) \\
\left.\quad-\frac{Z(s)}{2 U^{2}(x(s))}\right] d s \\
+\int_{0}^{t} \int_{\mathbb{Y}} e^{s}[\ln Q(x(s), u) \\
-Q(x(s), u)+1] \lambda(d u) d s,
\end{gathered}
$$

where

$$
\begin{gathered}
M_{1}(t)=\int_{0}^{t} \frac{e^{s}}{U(x(s))} \sum_{i, j=1}^{n} \sigma_{i j}(s) x_{i}(s) x_{j}^{\theta_{i j}}(s) d w_{j}(s), \\
M_{2}(t)=\int_{0}^{t} \int_{\mathbb{V}} e^{s} \ln Q(x(s-), u) \widetilde{N}(d s, d u)
\end{gathered}
$$

are two local martingales. By virtue of Lemma 6, for any $\alpha, \beta, T>0$ we have 


$$
\begin{array}{r}
P\left\{\omega: \sup _{0 \leq t \leq T}\left[M_{1}(t)+M_{2}(t)-\frac{\alpha}{2} \int_{0}^{t} \frac{e^{2 s} Z(s)}{U^{2}(x(s))} d s\right.\right. \\
-\frac{1}{\alpha} \int_{0}^{t} \int_{\mathbb{Y}}\left(Q^{\alpha e^{s}}(x(s), u)\right. \\
\left.-1-\alpha e^{s} \ln Q(x(s), u)\right) \\
\times \lambda(d u) d s] \geq \beta\} \leq e^{-\alpha \beta} .
\end{array}
$$

Choose $T=k, \alpha=\epsilon e^{-k}$, and $\beta=\left(2 e^{k} \ln k\right) / \epsilon$, where $k \in \mathbb{N}$, $0<\epsilon<1 / 2$, in the above equation. Since $\sum_{k=1}^{\infty} k^{-2}<\infty$, using the Borel-Cantelli lemma yields that there exists an $\Omega_{0} \subseteq \Omega$ with $P\left(\Omega_{0}\right)=1$ such that for any $\omega \in \Omega_{0}$ an integer $k_{0}=$ $k_{0}(\omega)>0$ can be found such that

$$
\begin{aligned}
& M_{1}(t)+M_{2}(t) \\
& \leq \frac{2 e^{k} \ln k}{\epsilon}+\frac{\epsilon e^{-k}}{2} \int_{0}^{t} \frac{e^{2 s} Z(s)}{U^{2}(x(s))} d s \\
& +\frac{1}{\epsilon e^{-k}} \int_{0}^{t} \int_{\mathbb{Y}}\left(Q^{\epsilon e^{s-k}}(x(s), u)-1\right. \\
& \left.\quad-\epsilon e^{s-k} \ln Q(x(s), u)-1\right) \lambda(d u) d s,
\end{aligned}
$$

for $0 \leq t \leq k$ and $k \geq k_{0}$. Thus (55) results in

$$
\begin{aligned}
& \ln U(x(t)) \\
& \leq e^{-t} \ln U(x(0))+\frac{2 e^{k-t} \ln k}{\epsilon} \\
& +\int_{0}^{t} e^{s-t}\left[\ln U(x(s))+\frac{1}{U(x(s))}\right. \\
& \times \sum_{i=1}^{n} x_{i}(s) r_{i}(s)-\frac{1}{U(x(s))} \\
& \times \sum_{i, j=1}^{n} x_{i}(s) a_{i j}(s) x_{j}^{\alpha_{i j}}(s) \\
& \left.-\frac{(1-\epsilon)}{2} \frac{Z(s)}{U^{2}(x(s))}\right] d s \\
& +\int_{0}^{t} \int_{\mathbb{Y}} e^{s-t}[\ln Q(x(s), u)-Q(x(s), u)+1] \lambda(d u) d s \\
& +\frac{1}{\epsilon e^{t-k}} \\
& \times \int_{0}^{t} \int_{\mathbb{Y}}\left(Q^{\epsilon e^{s-k}}(x(s), u)\right. \\
& \left.-1-\epsilon e^{s-k} \ln Q(x(s), u)\right) \lambda(d u) d s \\
& :=J_{1}(t)+J_{2}(t)+J_{3}(t)+J_{4}(t),
\end{aligned}
$$

for $0 \leq t \leq k$ and $k \geq k_{0}$. For any $x \in \mathbb{R}_{+}^{n}$ and $u \in \mathbb{Y}$, compute that

$$
\begin{aligned}
G(x(s)):= & \ln U(x(s))+\frac{1}{U(x(s))} \sum_{i=1}^{n} x_{i}(s) r_{i}(s) \\
& -\frac{1}{U(x(s))} \sum_{i, j=1}^{n} x_{i}(s) a_{i j}(s) x_{j}^{\alpha_{i j}}(s) \\
& -\frac{(1-\epsilon)}{2} \frac{Z(s)}{U^{2}(x(s))} \\
\leq & \ln U(x(s))+\sum_{i=1}^{n} r_{i}(s)-\left(\vec{a}_{i i}^{l}\right) \sum_{i=1}^{n} x_{i}^{\alpha_{i i}}(s) \\
& -\frac{(1-\epsilon)\left(\widetilde{\sigma}_{i i}^{l}\right)^{2}}{2} \sum_{i=1}^{n} x_{i}^{2 \theta_{i i}}(s) .
\end{aligned}
$$

When $\left(\widetilde{a}_{i i}^{l}\right)>0$ or $\left(\widetilde{\sigma}_{i i}^{l}\right)>0$, we obtain

$$
G(x(s)) \leq-K|x(s)|^{\alpha_{i i} \vee 2 \theta_{i i}}+o\left(|x(s)|^{\alpha_{i i} \vee 2 \theta_{i i}}\right) .
$$

Noting the inequality $\ln x \leq x-1, x>0$, we have

$$
\ln Q(x(s), u)-Q(x(s), u)+1 \leq 0 .
$$

In the light of Taylor's series expansion, for $\epsilon \in(0,1 / 2], x \in$ $\mathbb{R}_{+}^{n}, u \in \mathbb{Y}$, and $s \leq k$,

$$
\begin{aligned}
Q^{\epsilon e^{s-k}}(x, u)= & 1+\epsilon e^{s-k} \ln Q(x, u) \\
& +\frac{\epsilon^{2} e^{2(s-k)}}{2}(\ln Q(x, u))^{2} Q^{\xi}(x, u),
\end{aligned}
$$

where $\xi$ lies between 0 and $\epsilon$. Thus

$$
J_{4}(t)=\int_{0}^{t} \int_{\mathbb{Y}} \frac{\epsilon e^{2 s-t-k}}{2}(\ln Q(x, u))^{2} Q^{\xi}(x, u) \lambda(d u) d s .
$$

For $t \leq k$ and $k \geq k_{0}$, we have a decomposition for $J_{4}(t)$ as

$$
\begin{aligned}
J_{4}(t)= & \int_{0}^{t} \int_{0<Q(x, u)<1} \frac{\epsilon e^{2 s-t-k}}{2} \\
& \times(\ln Q(x, u))^{2} Q^{\xi}(x, u) \lambda(d u) d s \\
& +\int_{0}^{t} \int_{Q(x, u) \geq 1} \frac{\epsilon e^{2 s-t-k}}{2} \\
& \times(\ln Q(x, u))^{2} Q^{\xi}(x, u) \lambda(d u) d s \\
:= & \Gamma_{1}(t)+\Gamma_{2}(t) .
\end{aligned}
$$

For $0<Q(x, u)<1$ and $0 \leq \xi \leq \epsilon \leq 1 / 2$, we have $Q^{\xi}(x, u) \leq$ 1. Hence

$$
\begin{aligned}
\Gamma_{1}(t) & \leq \int_{0}^{t} \int_{0<Q(x, u)<1} \frac{\epsilon e^{2 s-t-k}}{2}(\ln Q(x, u))^{2} \lambda(d u) d s \\
& \leq \int_{0}^{t} \int_{\mathbb{Y}} \frac{\epsilon e^{2 s-t-k}}{2}(\ln Q(x, u))^{2} \lambda(d u) d s .
\end{aligned}
$$


On the other hand, recalling the fundamental inequality

$$
\ln x \leq 4\left(x^{1 / 4}-1\right) \quad \text { for } x \geq 1
$$

and observing $Q^{\xi}(x, u) \leq Q^{(1 / 2)}(x, u)$ for $Q(x, u) \geq 1$ and $0 \leq \xi \leq \epsilon \leq 1 / 2$, we have

$$
\begin{aligned}
\Gamma_{2}(t) & \leq 16 \int_{0}^{t} \int_{Q(x, u) \geq 1} \frac{\epsilon e^{2 s-t-k}}{2} Q(x, u) \lambda(d u) d s \\
& \leq 16 \int_{0}^{t} \int_{\mathbb{Y}} \frac{\epsilon e^{2 s-t-k}}{2} Q(x, u) \lambda(d u) d s .
\end{aligned}
$$

Consequently, for $t \leq k$ and $k \geq k_{0}$

$$
\begin{aligned}
J_{4}(t) \leq \frac{\epsilon}{2} \int_{0}^{t} \int_{\mathbb{V}} e^{s-t}[ & (\ln Q(x, u))^{2} \\
& +16 Q(x, u)] \lambda(d u) d s .
\end{aligned}
$$

Hence, substituting (60), (62), and (69) into (59), we arrive at

$$
\begin{gathered}
\ln U(x(t)) \leq e^{-t} \ln U(x(0))+\frac{2 e^{k-t} \ln k}{\epsilon} \\
+\int_{0}^{t} e^{s-t}\left[\ln U(x(s))+\sum_{i=1}^{n} r_{i}(s)\right. \\
-\left(\widetilde{a}_{i j}^{l}\right) \sum_{i=1}^{n} x_{i}^{\alpha_{i i}}(s) \\
\left.-\frac{(1-\epsilon)\left(\widetilde{\sigma}_{i j}^{l}\right)^{2}}{2} \sum_{i=1}^{n} x_{i}^{2 \theta_{i i}}(s)\right] d s \\
+\frac{\epsilon}{2} \int_{0}^{t} \int_{\mathbb{Y}} e^{s-t}\left[(\ln Q(x(s), u))^{2}\right. \\
+16 Q(x(s), u)] \lambda(d u) d s .
\end{gathered}
$$

Moreover, by the condition (52), we can deduce that for $x \in$ $\mathbb{R}_{+}^{n}$ and $\delta \in\left(0, \alpha_{i i} \wedge 2 \theta_{i i}\right]$

$$
\begin{gathered}
\int_{\mathbb{Y}}\left[(\ln Q(x, u))^{2}+16 Q(x, u)\right] \lambda(d u) \\
\leq K|x|^{\delta}+o\left(|x|^{\delta}\right) .
\end{gathered}
$$

Thus, combining (61), (62), and (69) with (71), when $\left(\vec{a}_{i i}^{l}\right)>0$ or $\left(\widetilde{\sigma}_{i i}^{l}\right)>0$, there exists a constant $K$ such that

$$
J_{2}(t)+J_{3}(t)+J_{4}(t) \leq K \int_{0}^{t} e^{s-t} d s \leq K .
$$

Hence, for any $\omega \in \Omega_{0}, 0 \leq t \leq k$, and $k \geq k_{0}$,

$$
\ln U(x(t)) \leq e^{-t} \ln U(x(0))+\frac{2 e^{k-t} \ln k}{\epsilon}+K .
$$

In particular, for $k-1 \leq t \leq k$ and $k \geq k_{0}+1$,

$$
\begin{aligned}
& \frac{\ln U(x(t))}{\ln t} \\
& \quad \leq \frac{e^{-t} \ln U(x(0))+K}{\ln (k-1)}+\frac{2 e \ln k}{\epsilon \ln (k-1)} \text { a.s., }
\end{aligned}
$$

which implies that

$$
\limsup _{t \rightarrow \infty} \frac{\ln U(x(t))}{\ln t} \leq \frac{2 e}{\epsilon} \quad \text { a.s. }
$$

The desired assertion then follows by letting $\epsilon \uparrow 1 / 2$ and $|x| \leq$ $U(x)$.

Noting the limit $\lim _{t \rightarrow \infty}(\ln t / t)=0$, we can deduce from Theorem 7 that the sample Lyapunov exponent of (4) is less than or equal to zero, which is stated in the following corollary.

Corollary 8. Under conditions of Theorem 7, one has

$$
\limsup _{t \rightarrow \infty} \frac{\ln (|x(t)|)}{t} \leq 0 \quad \text { a.s. }
$$

\section{Extinction and Nonpersistence}

One of the important problems in population biology is whether all species of one system will become extinct over time. There are many factors including natural and manmade factors which may cause the extinction of some species, such as low birth rate, high death rate, decreasing habitats, and aggravating living environment. Sometimes, small even large population of some species may be destroyed by some extraordinary perturbation. To proceed, we need some appropriate definitions of extinction and persistence. Hallam and Ma proposed the concepts of weak persistence [22], weak persistence in the mean [23], and strong persistence in the mean [24] for some deterministic models. Liu and Wang $[25,26]$ applied thes concepts to stochastic logistic models. In this section, we show, that if the noise is sufficiently large, the solution of the system (4) will become extinct with probability one, nonpersistent in time average, or weakly persistent.

Definition 9. Stochastic population dynamics (4) is said to be extinct with probability 1 if, for every initial datum $x_{0} \in \mathbb{R}_{+}^{n}$, the solution $x(t), t \geq 0$, has the property

$$
\lim _{t \rightarrow \infty} x(t)=0 \quad \text { a.s. }
$$

Definition 10. The population $x(t)$ is said to be nonpersistent in time average with probability 1 if $\limsup _{t \rightarrow \infty}(1 /$ t) $\int_{0}^{t} x(s) d s=0$ a.s.

Definition 11. The population $x(t)$ is said to be weakly persistent with probability 1 if $\lim _{\sup } \rightarrow \operatorname{si\infty } x(t)>0$ a.s. 
Theorem 12. Let conditions of Theorem 2 hold. If $\min _{1 \leq j \leq n} \theta_{i j}=$ 0 for some $i, i=1, \ldots, n$, and

$$
\begin{aligned}
& \limsup _{t \rightarrow \infty} \frac{1}{t} \int_{0}^{t}\left(r_{i}(s)-\frac{1}{2} \sum_{j}^{n} \sigma_{i j}^{2}(s) I_{\left\{\theta_{i j}=0\right\}}\right) d s \\
& =b^{*}<0 \quad \text { a.s. }
\end{aligned}
$$

hold, then ith population $x_{i}(t)$ will become extinct with probability 1.

Proof. Applying Itô's formula to $\ln x_{i}(t)$ and integrating 0 into $t$ yield

$$
\begin{aligned}
\ln x_{i}(t) \leq & \ln x_{i}(0) \\
& +\int_{0}^{t}\left[r_{i}(s)-\frac{1}{2} \sum_{j}^{n} \sigma_{i j}^{2}(s) I_{\left\{\theta_{i j}=0\right\}}\right. \\
& \left.-\frac{1}{2} \sum_{j}^{n} \sigma_{i j}^{2}(s) x_{j}^{2 \theta_{i j}}(s) I_{\left\{\theta_{i j} \neq 0\right\}}\right] d s \\
& -\left(a_{i i}^{l}\right) \int_{0}^{t} x_{i}^{\alpha_{i i}}(s) d s \\
& +\int_{0}^{t} \int_{\mathbb{Y}}\left(\ln \left(1+H_{i}(x(s), u)\right)\right. \\
& +M_{1 i}(t)+M_{2 i}(t)+M_{3 i}(t),
\end{aligned}
$$

where

$$
\begin{gathered}
M_{1 i}(t)=\int_{0}^{t} \sum_{j=1}^{n} \sigma_{i j}(s) I_{\left\{\theta_{i j}=0\right\}} d w_{j}(s), \\
M_{2 i}(t)=\int_{0}^{t} \sum_{j=1}^{n} \sigma_{i j}(s) x_{j}^{\theta_{i j}}(s) I_{\left\{\theta_{i j} \neq 0\right\}} d w_{j}(s), \\
M_{3 i}(t)=\int_{0}^{t} \int_{\mathbb{Y}} \ln \left(1+H_{i}(x(s-), u)\right) \widetilde{N}(d s, d u)
\end{gathered}
$$

are the local martingales with the quadratic variations

$$
\begin{gathered}
\left\langle M_{1 i}\right\rangle_{t}=\int_{0}^{t} \sum_{j=1}^{n} \sigma_{i j}^{2}(s) I_{\left\{\theta_{i j}=0\right\}} d s \\
\left\langle M_{2 i}\right\rangle_{t}=\int_{0}^{t} \sum_{j=1}^{n} \sigma_{i j}^{2}(s) x_{j}^{2 \theta_{i j}}(s) I_{\left\{\theta_{i j} \neq 0\right\}} d s, \\
\left\langle M_{3 i}\right\rangle_{t}=\int_{0}^{t} \int_{\mathbb{Y}}\left(\ln \left(1+H_{i}(x(s), u)\right)\right)^{2} \lambda(d u) d s .
\end{gathered}
$$

Since $\left\langle M_{1 i}\right\rangle_{t} \leq n\left(\bar{\sigma}_{i j}^{u}\right)^{2} t$, by the strong law of large numbers for martingales, we have that $\lim _{t \rightarrow \infty}\left(M_{1 i}(t) / t\right)=0$ a.s.
On the other hand, for every integer $k \geq 1$, by using the exponential martingale inequality, we have

$$
\begin{aligned}
& P\left\{\sup _{0 \leq t \leq n}[\right. M_{2 i}(t)+M_{3 i}(t) \\
&-\frac{1}{2} \int_{0}^{t} \sum_{j=1}^{n} \sigma_{i j}^{2}(s) x_{j}^{2 \theta_{i j}}(s) I_{\left\{\theta_{i j} \neq 0\right\}} d s \\
&-\int_{0}^{t} \int_{\mathbb{Y}}\left(H_{i}(x(s), u)\right. \\
&\geq 2 \ln k\} \leq \frac{1}{k^{2}} .
\end{aligned}
$$

It then follows from the Borel-Cantelli lemma that for almost all $\omega \in \Omega$, there is a random integer $k_{0}=k_{0}(\omega)$ such that, for $k \geq k_{0}$,

$$
\begin{aligned}
& M_{2 i}(t)+M_{3 i}(t) \\
& \leq 2 \ln k+\frac{1}{2} \int_{0}^{t} \sum_{j=1}^{n} \sigma_{i j}^{2}(s) x_{j}^{2 \theta_{i j}}(s) I_{\left\{\theta_{i j} \neq 0\right\}} d s \\
& \quad+\int_{0}^{t} \int_{\mathbb{Y}}\left(H_{i}(x(s), u)\right. \\
& \left.\quad-\ln \left(1+H_{i}(x(s), u)\right)\right) \lambda(d u) d s .
\end{aligned}
$$

Substituting this inequality into (79) gives

$$
\begin{aligned}
\ln x_{i}(t) \leq & \ln x_{i}(0)+2 \ln k \\
& +\int_{0}^{t}\left(r_{i}(s)-\frac{1}{2} \sum_{j}^{n} \sigma_{i j}^{2}(s) I_{\left\{\theta_{i j}=0\right\}}\right) d s \\
& -\left(a_{i i}^{l}\right) \int_{0}^{t} x_{i}^{\alpha_{i i}}(s) d s+M_{1 i}(t) .
\end{aligned}
$$

Consequently for $0<k-1 \leq t \leq k, k \geq k_{0}$,

$$
\begin{aligned}
\frac{\ln x_{i}(t)}{t} \leq & \frac{\ln x_{i}(0)}{t}+\frac{2 \ln k}{k-1} \\
& +\frac{1}{t} \int_{0}^{t}\left(r_{i}(s)-\frac{1}{2} \sum_{j}^{n} \sigma_{i j}^{2}(s) I_{\left\{\theta_{i j}=0\right\}}\right) d s \\
& +\frac{M_{1 i}(t)}{t} .
\end{aligned}
$$

Taking superior limit on both sides and using (78), one can see that

$$
\limsup _{t \rightarrow \infty} \frac{\ln x_{i}(t)}{t} \leq b^{*}<0
$$


Therefore $\lim _{t \rightarrow \infty} x_{i}(t)=0$ a.s.; that is, $i$ th population will become extinct with probability 1 .

Theorem 13. Let conditions of Theorem 2 hold. If $a_{i i}^{l}>0, \alpha_{i i} \geq$ 1 , and

$$
\limsup _{t \rightarrow \infty} \frac{1}{t} \int_{0}^{t}\left(r_{i}(s)-\frac{1}{2} \sum_{j}^{n} \sigma_{i j}^{2}(s) I_{\left\{\theta_{i j}=0\right\}}\right) d s=0 \quad \text { a.s., }
$$

for some $i=1,2, \ldots, n$, then ith population of system (4) is nompersistent in the time average with probability 1.

Theorem 14. Let conditions of Theorem 2 hold. If $a_{i i}^{l}>0, \alpha_{i i} \geq$ 1 , and

$$
\limsup _{t \rightarrow \infty} \frac{1}{t} \int_{0}^{t}\left(r_{i}(s)-\frac{1}{2} \sum_{j}^{n} \sigma_{i j}^{2}(s) I_{\left\{\theta_{i j}=0\right\}}\right) d s>0 \quad \text { a.s., }
$$

for some $i=1,2, \ldots, n$, then ith population of system (4) is weakly persistent with probability 1 .

The proofs of Theorems 13 and 14 are similar to those of $[14$, Theorem $6.3,6.4]$, so we omit them here.

\section{Examples and Simulations}

In this section we provide a numerical example to substantiate the analytical findings for the stochastic model system reported in the previous sections.

Example 1. Consider the two-species nonautonomous stochastic Gilpin-Ayala system with jumps

$$
\begin{aligned}
d x_{1}(t)= & x_{1}(t)\left(r_{1}(t)-a_{11}(t) x_{1}^{\alpha_{11}}(t)-a_{12}(t) x_{2}^{\alpha_{12}}(t)\right) d t \\
& +\sigma_{11}(t) x_{1}^{1+\theta_{11}}(t) d w_{1}(t) \\
& +\sigma_{12}(t) x_{1}(t) x_{2}^{\theta_{12}}(t) d w_{2}(t) \\
& +\int_{\mathbb{V}} x_{1}(t) H_{1}(x(t-), u) \widetilde{N}(d t, d u), \\
d x_{2}(t)= & x_{2}(t)\left(r_{2}(t)-a_{21}(t) x_{1}^{\alpha_{21}}(t)-a_{22}(t) x_{2}^{\alpha_{22}}(t)\right) d t \\
& +\sigma_{21}(t) x_{1}^{\theta_{21}}(t) x_{2}(t) d w_{1}(t) \\
& +\sigma_{22}(t) x_{2}^{1+\theta_{22}}(t) d w_{2}(t) \\
& +\int_{\mathbb{V}} x_{2}(t) H_{2}(x(t-), u) \widetilde{N}(d t, d u),
\end{aligned}
$$

where $x_{1}(t)$ and $x_{2}(t)$ represent the population sizes of species.

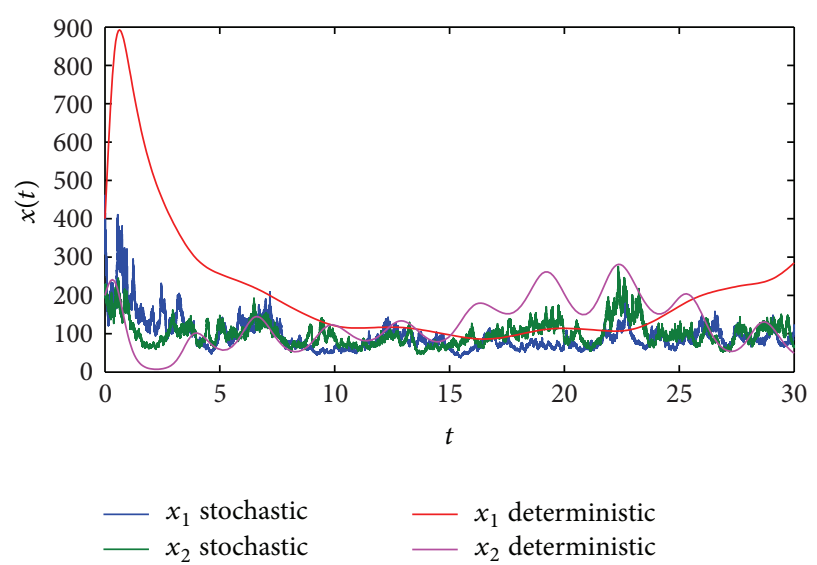

FIGURE 1: Solutions of (89) with parameters (90) and initial data $x_{1}(0)=400, x_{2}(0)=200, \mathbb{Y}=(0,+\infty)$, and $\lambda(\mathbb{Y})=1$.

Let

$$
\begin{gathered}
r_{1}(t)=1.5+\frac{3}{1+t^{2}}, \quad r_{2}(t)=\sqrt{6+\frac{4}{2+\sin t}}, \\
a_{11}(t)=0.01\left(0.4+\sin ^{2} \frac{t}{10}\right), \\
a_{12}(t)=0.1 \sin ^{2} \frac{t}{2} \\
a_{21}(t)=0.07 \sin ^{2} t \\
a_{22}(t)=0.015(1-0.4 \cos (0.3 t)), \\
\alpha_{11}=1, \quad \alpha_{12}=\frac{1}{4}, \\
\alpha_{21}=\frac{2}{3}, \quad \alpha_{22}=1 \\
\sigma_{11}(t)=0.001\left(1+\frac{1}{\sqrt{4+t}}\right) \\
\sigma_{22}(t)=0.01 \ln \left(2+\frac{1}{1+t}\right) \\
\sigma_{12}(t)=\sigma_{21}(t)=0 \\
\theta_{11}(t)=\frac{1}{2}, \quad \theta_{12}(t)=\theta_{21}(t)=0 \\
5 \\
\theta_{22}(t)=\frac{4}{5} . \\
\end{gathered}
$$

If we choose $H_{1}(x(t-), u)=x_{1}(t)$ and $H_{2}(x(t-), u)=x_{2}(t)$, it is easy to check that the assumptions (H1) and (H2) hold. Hence, from Theorem 2, there exists a global solution of (89) with any initial value $\left(x_{1}(0), x_{2}(0)\right)$. Figure 1 confirms this.

In Figure 2, we aimed to illustrate the effect of environmental noise on the extinction of species. We choose $r_{1}(t)=r_{2}(t)=1+\left(3 /\left(1+t^{2}\right)\right), \sigma_{11}(t)=1.45-(1 / \sqrt{1+t})$, $\sigma_{22}(t)=0.1(1-(1 / \sqrt{1+t}))$, and $\theta_{11}(t)=0, \theta_{22}(t)=1 / 2$, 


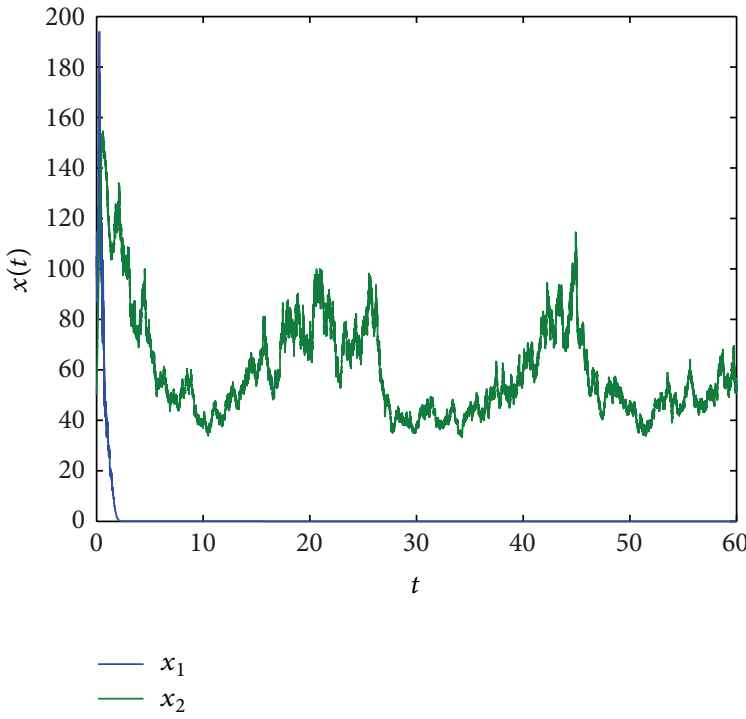

(a)

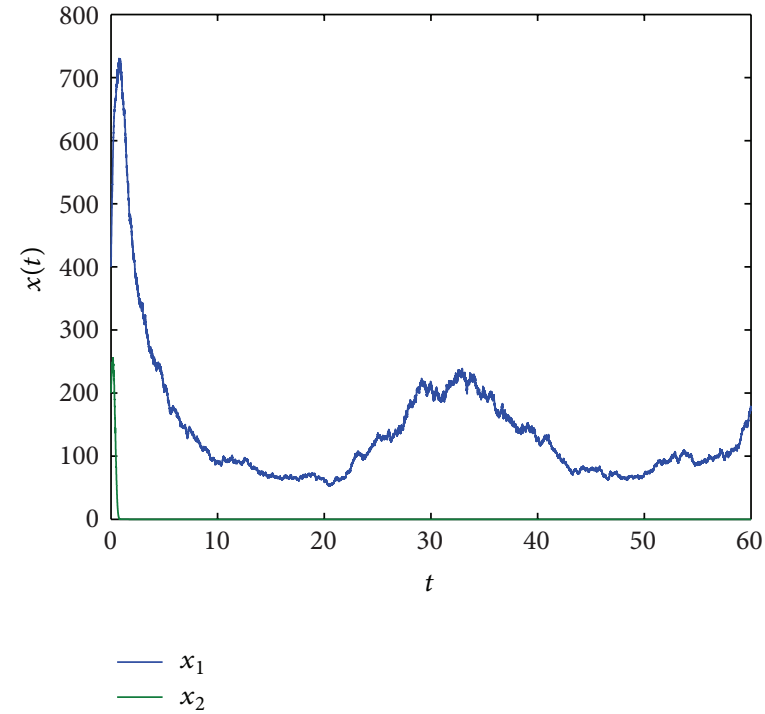

(b)

Figure 2: Solutions of systems (89) for $r_{1}(t)=r_{2}(t)=1+\left(3 /\left(1+t^{2}\right)\right), \sigma_{11}(t)=1.45-(1 / \sqrt{1+t}), \sigma_{22}(t)=0.1(1-(1 / \sqrt{1+t})), \theta_{11}(t)=0$, and $\theta_{22}(t)=1 / 2$; initial data $x_{1}(0)=100, x_{2}(0)=50(\mathrm{a}), r_{1}(t)=1.5+\sin t+\left(1 /\left(1+t^{2}\right)\right), r_{2}(t)=1.5+\left(2 /\left(1+t^{2}\right)\right), \sigma_{11}(t)=1.8-(1 / \sqrt{1+t})$, $\sigma_{22}(t)=1.8-(1 /(1+t)), \theta_{11}(t)=1$, and $\theta_{22}(t)=0$ initial data $x_{1}(0)=400, x_{2}(0)=200(\mathrm{~b})$.

and the rest of parameters are the same as those in Figure 1. Compute

$$
\limsup _{t \rightarrow \infty} \frac{1}{t} \int_{0}^{t}\left[r_{1}(s)-\frac{1}{2} \sigma_{11}^{2}(s)\right] d s \approx-0.042119 .
$$

In view of Theorem 12, it follows that the first population will become extinct with probability one, as demonstrated in Figure 2(a). If we change the coefficients with

$$
\begin{gathered}
r_{1}(t)=1.5+\sin t+\frac{1}{1+t^{2}}, \\
r_{2}(t)=1.5+\frac{2}{1+t^{2}}, \\
\sigma_{11}(t)=1.8-\frac{1}{\sqrt{1+t}}, \\
\sigma_{22}(t)=1.8-\frac{1}{1+t},
\end{gathered}
$$

and $\theta_{11}(t)=1, \theta_{22}(t)=0$, we can get by the computer that

$$
\limsup _{t \rightarrow \infty} \frac{1}{t} \int_{0}^{t}\left[r_{2}(s)-\frac{1}{2} \sigma_{22}^{2}(s)\right] d s \approx-0.12 .
$$

Hence, according to Theorem 12, the second species will become extinct with probability one, which is illustrated in Figure 2(b).

\section{Conclusions}

This paper is concerned with a nonautonomous stochastic Gilpin-Ayala competition model with jumps. We show that the model (4) admits a unique global positive solution. Several moment and pathwise asymptotic estimators are obtained. Moreover, we discuss the extinction and nonpersistence of each population. Three computer simulations are presented which support the theoretical results.

\section{Acknowledgments}

This work was substantially supported by the National Natural Sciences Foundation of China (nos. 11071259, 11371374) and Research Fund for the Doctoral Program of Higher Education of China (no. 20110162110060).

\section{References}

[1] A. Lotka, Elements of Physical Biology, Williams and Wilkins, Baltimore, Md, USA, 1924

[2] V. Volterra, "Variazioni a fluttuazioni del numero d'individui in specie d'animali conviventi," Memorie della R. Accademia dei Lincei, vol. 2, pp. 31-113, 1926.

[3] K. Gopalsamy, Stability and Oscillations in Delay Differential Equations of Population Dynamics, vol. 74 of Mathematics and Its Applications, Kluwer Academic Publishers, Dordrecht, The Netherlands, 1992.

[4] Y. Kuang, Delay Differential Equations with Applications in Population Dynamics, vol. 191 of Mathematics in Science and Engineering, Academic Press, Boston, Mass, USA, 1993.

[5] M. Liao, X. Tang, and C. Xu, "Dynamics of a competitive LotkaVolterra system with three delays," Applied Mathematics and Computation, vol. 217, no. 24, pp. 10024-10034, 2011.

[6] D. Xiao and W. Li, "Limit cycles for the competitive three dimensional Lotka-Volterra system," Journal of Differential Equations, vol. 164, no. 1, pp. 1-15, 2000. 
[7] M. E. Gilpin and F. J. Ayala, "Global models of growth and competition," Proceedings of the National Academy of Sciences of the United States of America, vol. 70, pp. 3590-3593, 1973.

[8] M. Fan and K. Wang, "Global periodic solutions of a generalized $n$-species Gilpin-Ayala competition model," Computers \& Mathematics with Applications, vol. 40, no. 10-11, pp. 1141-1151, 2000.

[9] M. He, Z. Li, and F. Chen, "Permanence, extinction and global attractivity of the periodic Gilpin-Ayala competition system with impulses," Nonlinear Analysis: Real World Applications, vol. 11, no. 3, pp. 1537-1551, 2010.

[10] J. Bao, X. Mao, G. Yin, and C. Yuan, "Competitive LotkaVolterra population dynamics with jumps," Nonlinear Analysis: Theory, Methods \& Applications, vol. 74, no. 17, pp. 6601-6616, 2011.

[11] A. Bahar and X. Mao, "Stochastic delay Lotka-Volterra model," Journal of Mathematical Analysis and Applications, vol. 292, no. 2, pp. 364-380, 2004.

[12] X. Li and X. Mao, "Population dynamical behavior of nonautonomous Lotka-Volterra competitive system with random perturbation," Discrete and Continuous Dynamical Systems. Series A, vol. 24, no. 2, pp. 523-545, 2009.

[13] X. Mao, S. Sabanis, and E. Renshaw, "Asymptotic behaviour of the stochastic Lotka-Volterra model," Journal of Mathematical Analysis and Applications, vol. 287, no. 1, pp. 141-156, 2003.

[14] M. Jovanović and M. Vasilova, "Dynamics of non-autonomous stochastic Gilpin-Ayala competition model with time-varying delays," Applied Mathematics and Computation, vol. 219, no. 12, pp. 6946-6964, 2013.

[15] M. Vasilova and M. Jovanović, "Dynamics of Gilpin-Ayala competition model with random perturbation," Filomat, vol. 24, no. 1, pp. 101-113, 2010.

[16] M. Vasilova and M. Jovanović, "Stochastic Gilpin-Ayala competition model with infinite delay," Applied Mathematics and Computation, vol. 217, no. 10, pp. 4944-4959, 2011.

[17] J. Bao and C. Yuan, "Stochastic population dynamics driven by Lévy noise," Journal of Mathematical Analysis and Applications, vol. 391, no. 2, pp. 363-375, 2012.

[18] X. Zhang and K. Wang, "Asymptotic behavior of stochastic Gilpin-Ayala mutualism model with jumps," Electronic Journal of Diffrential Equations, vol. 162, pp. 1-17, 2013.

[19] X. Mao, Stochastic Differential Equations and Applications, Horwood, Chichester, UK, 2nd edition, 2008.

[20] I.-S. Wee, "Stability for multidimensional jump-diffusion processes," Stochastic Processes and their Applications, vol. 80, no. 2, pp. 193-209, 1999.

[21] D. Applebaum, Lévy Processes and Stochastic Calculus, vol. 116 of Cambridge Studies in Advanced Mathematics, Cambridge University Press, Cambridge, UK, Second edition, 2009.

[22] T. G. Hallam and Z. E. Ma, "Persistence in population models with demographic fluctuations," Journal of Mathematical Biology, vol. 24, no. 3, pp. 327-339, 1986.

[23] Z. E. Ma, G. R. Cui, and W. D. Wang, "Persistence and extinction of a population in a polluted environment," Mathematical Biosciences, vol. 101, no. 1, pp. 75-97, 1990.

[24] Z. E. Ma and T. G. Hallam, "Effects of parameter fluctuations on community survival," Mathematical Biosciences, vol. 86, no. 1, pp. 35-49, 1987.

[25] M. Liu and K. Wang, "Survival analysis of stochastic singlespecies population models in polluted environments," Ecological Modelling, vol. 220, no. 9-10, pp. 1347-1357, 2009.
[26] M. Liu and K. Wang, "Persistence and extinction in stochastic non-autonomous logistic systems," Journal of Mathematical Analysis and Applications, vol. 375, no. 2, pp. 443-457, 2011. 


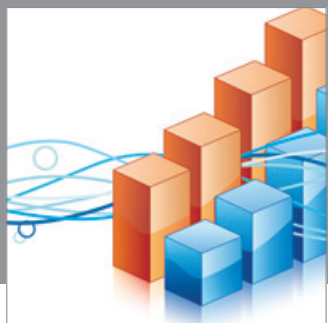

Advances in

Operations Research

mansans

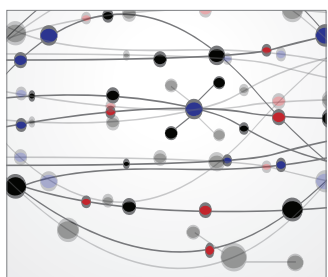

The Scientific World Journal
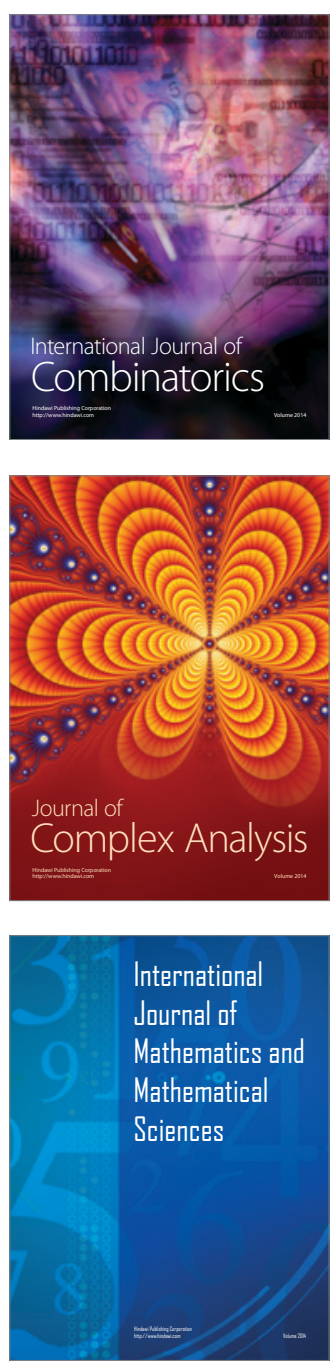
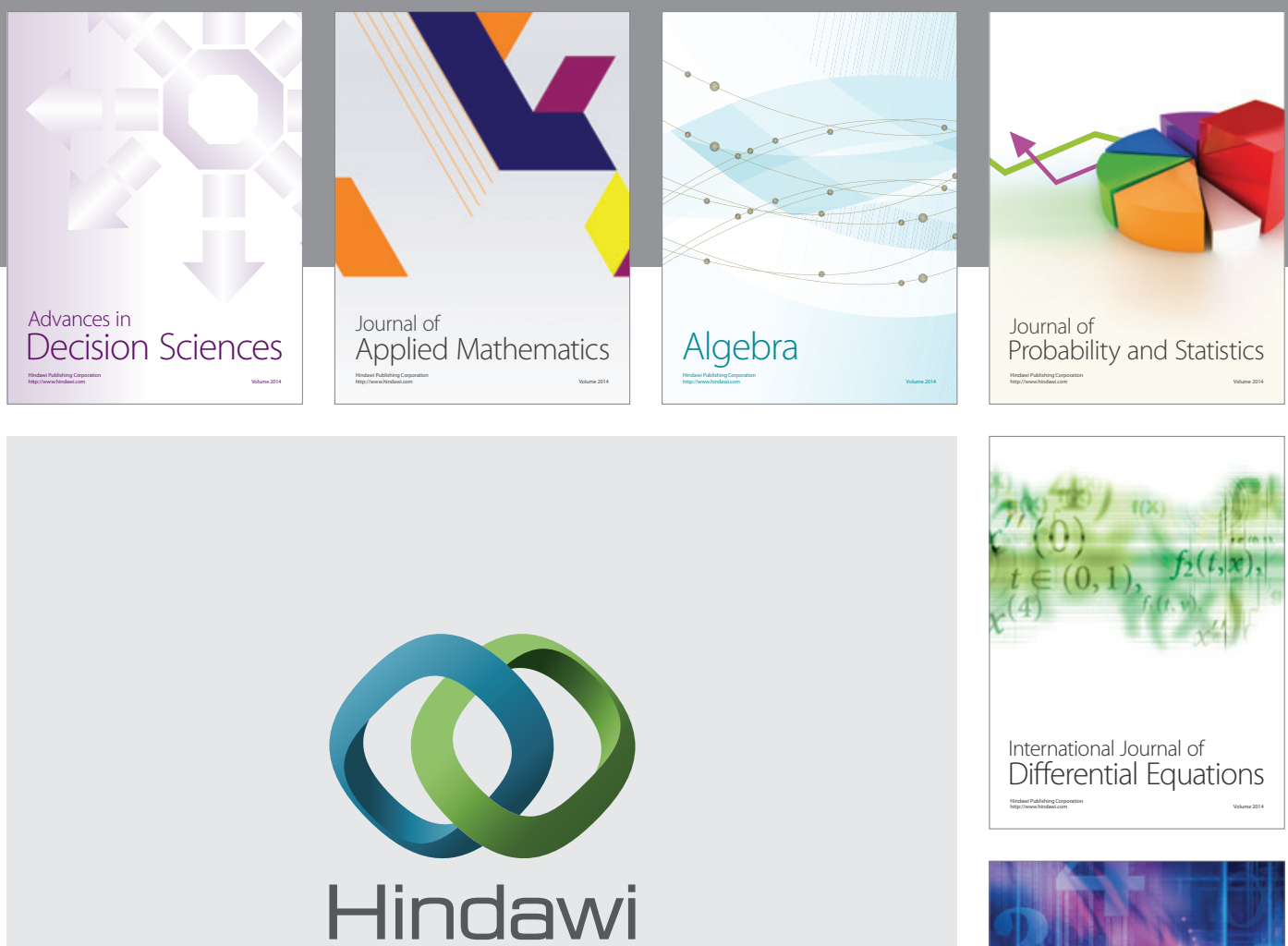

Submit your manuscripts at http://www.hindawi.com
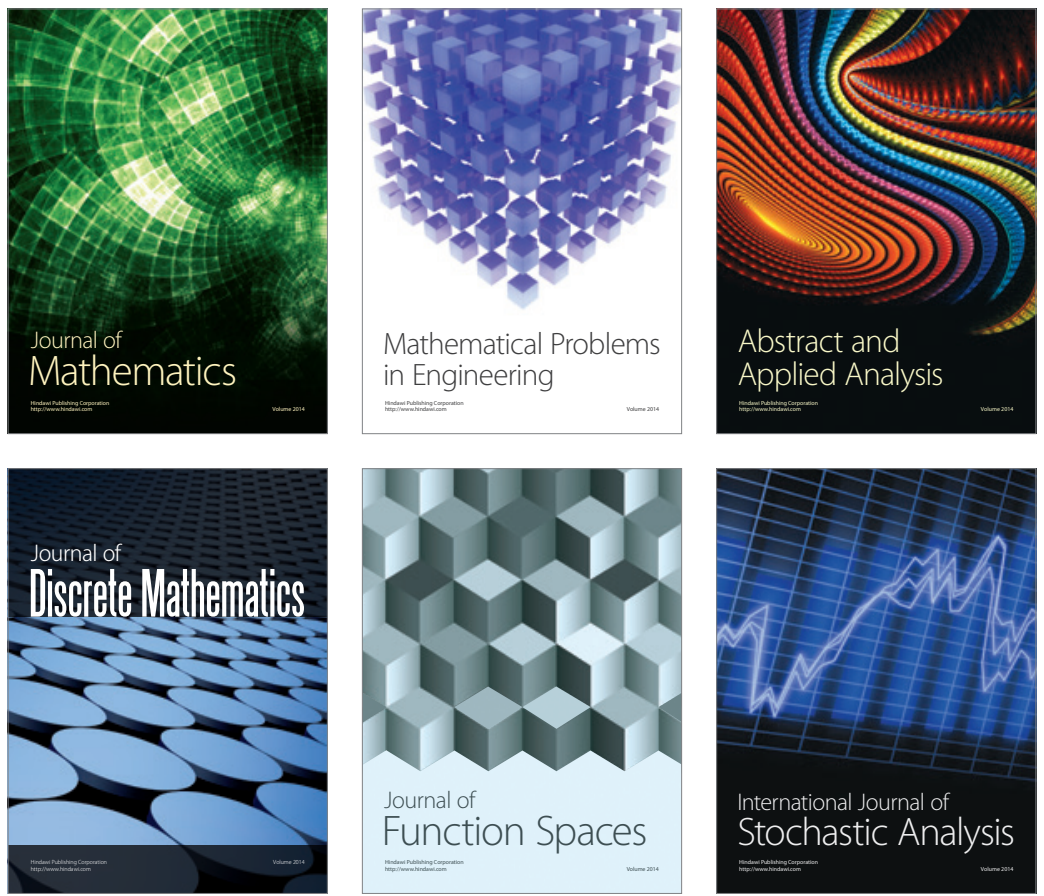

Journal of

Function Spaces

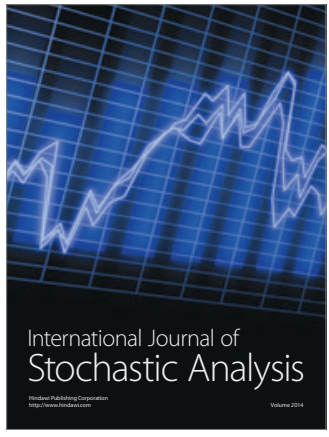

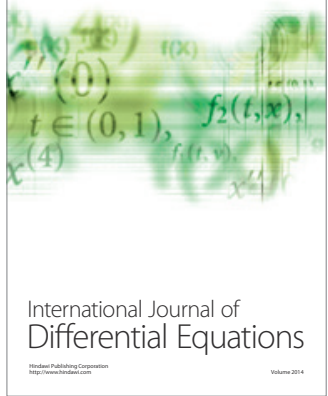
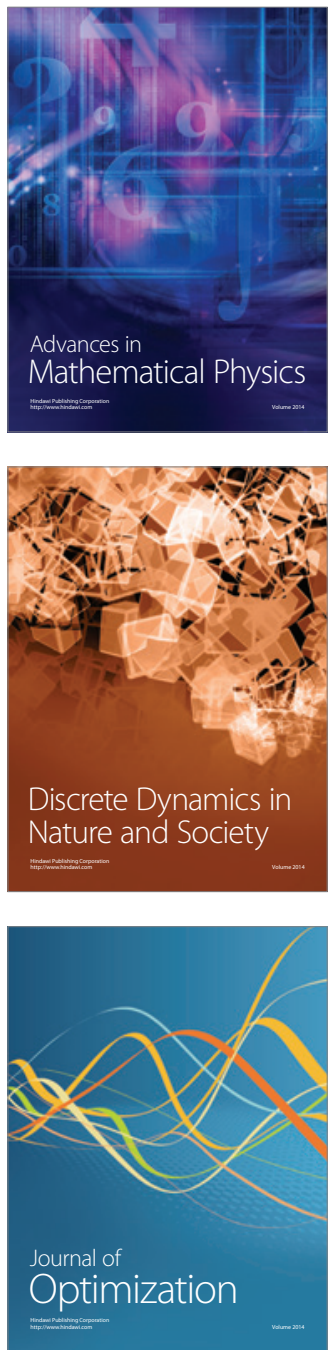